\title{
Genetic Deletion of Rheb1 in the Brain Reduces Food Intake and Causes Hypoglycemia with Altered Peripheral Metabolism
}

Wanchun Yang ${ }^{1, \dagger}$, Wanxiang Jiang ${ }^{1, \dagger}$, Liping Luo ${ }^{1}$, Jicheng Bu ${ }^{1}$, Dejiang Pang ${ }^{2}$, Jing Wei ${ }^{1}$, Chongyangzi Du ${ }^{1}$, Xiaoqiang Xia ${ }^{1}$, Yiyuan Cui ${ }^{1}$, Shuang Liu ${ }^{3}$, Qing Mao ${ }^{3, *}$ and Mina Chen ${ }^{1, *}$

1 Laboratory of Molecular Neurobiology, State Key Laboratory of Biotherapy/West China Hospital, Sichuan University, Chengdu 610041, China; E-Mails: yangwanchunscu@gmail.com (W.Y.); wanxjiang@gmail.com (W.J.); lipingluo1987@gmail.com (L.L.); jichengbu@gmail.com (J.B.); weijing3131@gmail.com (J.W.); duchongyangzi@gmail.com (C.D.); xiaoqiangxia@gmail.com (X.X.); cuiyy307@gmail.com (Y.C.)

2 West China School of Preclinical and Forensic Medicine, Sichuan University, Chengdu 610041, China; E-Mail: pangdejiang@gmail.com

3 Department of Neurosurgery, West China Hospital, Sichuan University, Chengdu 610041, China; E-Mail: liushuang3302@163.com

$\dagger$ These authors contributed equally to this work.

* Authors to whom correspondence should be addressed;

E-Mails: qingmao2000@163.com (Q.M.); chenmina2010@gmail.com (M.C.);

Tel.: +86-28-8516-4097 (M.C.); Fax: +86-28-8516-4095 (M.C.).

Received: 16 November 2013; in revised form: 12 December 2013 / Accepted: 7 January 2014 / Published: 21 January 2014

\begin{abstract}
Excessive food/energy intake is linked to obesity and metabolic disorders, such as diabetes. The hypothalamus in the brain plays a critical role in the control of food intake and peripheral metabolism. The signaling pathways in hypothalamic neurons that regulate food intake and peripheral metabolism need to be better understood for developing pharmacological interventions to manage eating behavior and obesity. Mammalian target of rapamycin ( $m T O R)$, a serine/threonine kinase, is a master regulator of cellular metabolism in different cell types. Pharmacological manipulations of $m T O R$ complex 1 (mTORCl) activity in hypothalamic neurons alter food intake and body weight. Our previous study identified Rhebl (Ras homolog enriched in brain 1) as an essential activator of $m T O R C l$ activity in the brain. Here we examine whether central Rhebl regulates food intake and peripheral metabolism through mTORC1 signaling. We find that genetic
\end{abstract}


deletion of Rheb1 in the brain causes a reduction in mTORCl activity and impairs normal food intake. As a result, Rhebl knockout mice exhibit hypoglycemia and increased lipid mobilization in adipose tissue and ketogenesis in the liver. Our work highlights the importance of central Rhebl signaling in euglycemia and energy homeostasis in animals.

Keywords: Rheb1; genetic deletion; food intake; hypoglycemia; ketogenesis

Abbreviations: $m$ TOR, mammalian target of rapamycin; $m T O R C 1$, mammalian target of rapamycin complex 1; mTORC2, mammalian target of rapamycin complex 2; Rheb1, Ras homolog enriched in brain 1; ARC, arcuate; POMC, proopiomelanocortin; DN, dominant negative; CA, constitutively active; Rhebl ko, Rhebl f/f Nestin-cre; HMG-CoA, hydroxy-methyl-glutaryl coenzyme A; HMGCS2, HMG-CoA synthase 2; HMGCL, HMG-CoA lyase; $A T G L$, adipose triglyceride lipase.

\section{Introduction}

Energy homeostasis is a fundamental mechanism used by multicellular organisms to maintain their survival and body growth [1]. It is tightly linked to food intake [2,3]. How the link between food intake and energy homeostasis is controlled remains incompletely understood. Recent studies show that central mammalian target of rapamycin ( $m T O R)$ signaling plays a critical role in the control of food intake and energy homeostasis [4-6]. $m T O R$, a serine/threonine kinase, is a master regulator of cellular metabolism, including protein and lipid synthesis, and energy production [7-9]. mTOR kinase functions in two distinct protein complexes-mTORC1 (mammalian target of rapamycin complex 1) and mTORC2 (mammalian target of rapamycin complex 2) [10,11]. mTORC1 activity is activated by growth factors (e.g., insulin) and nutrients (e.g., amino acids) [12], and negatively regulated by GTPase activity of the TSC (tuberous sclerosis complex) protein complex encoded by the TSC1 and TSC2 genes [13]. In 2006, a landmark paper showed that altered mTORC1 activity in hypothalamic neurons alters food intake and energy metabolism [4]. Intracerebroventricular injection of leucine in the vicinity of the arcuate (ARC) neurons in hypothalamus activates $m$ TORC1 activity and reduces food intake. Conversely, injection of $m T O R C 1$ inhibitor rapamycin increases food intake and blunts leptin-mediated anorectic effect [4]. Subsequently, S6 kinase $(S 6 K)$, a downstream substrate of $m T O R C 1$, is reported to regulate food intake and energy homeostasis [14]. The injection of adenovirus expressing dominant negative (DN) $S 6 K$ in the mediobasal hypothalamus increases food intake, while expressing constitutively active (CA) $S 6 K$ decreases food intake [14]. These studies establish the central control of food intake and energy homeostasis through hypothalamic neurons. However, a recent study shows that genetic deletion of TSC1 in hypothalamic POMC (proopiomelanocortin) neurons increases mTORCl activity, and food intake by TSCl knockout mice is increased and associated with increased body weight. Also, intracerebral rapamycin infusion was shown to reduce food intake and body weight in aged mice [15]. These results contradict earlier reports showing that increased $m$ TORCl activity in hypothalamic neurons reduces food intake. Although these studies have demonstrated a critical role of $m$ TORC1 activity in the control of food intake and energy metabolism, most of them are based on pharmacological or viral mediated manipulations of mTORCl activity 
in vivo. Therefore, the role of the $m T O R$ signaling pathway in the control of food intake and energy metabolism needs further clarification in genetic models. Our previous study shows that Rhebl (Ras homolog enriched in brain 1) is required for the activation of mTORC1 activity in the brain [16]. The genetic deletion of Rhebl in neural stem/progenitor cells of the brain (Rhebl f/f Nestin-cre, hereafter Rhebl ko) renders the mTORCl activity undetectable as shown by the absence of phosphorylation of $S 6$, whereas $m T O R C 2$ activity is not impaired [16]. The Rheb1 ko mice show impaired postnatal growth and reduced body weight by $64.7 \%$ at 3 -week-old, living for about 6 weeks [16]. The reason for the premature death is unknown. Now we show that the Rhebl ko mouse reduces food intake and develops severe hypoglycemia and peripheral metabolic adaptations to meet the energy demands of the brain.

\section{Results and Discussion}

\subsection{Genetic Deletion of Rheb1 in the Brain Disrupts Food Intake}

To examine the role of Rheb1/mTORC1 signaling in the regulation of food intake and energy homeostasis, we examined the mTORCl activity in Rhebl ko mice (Rhebl f/f, Nestin-cre) in which Rhebl was deleted by Cre activity expressed by Nestin promoter [17]. We focused on the hypothalamus, because it is critical in the central control of food intake. The results revealed that mTORCl activity was dramatically reduced in the hypothalamus of Rhebl ko, as indicated by pS6 (Ser240/244) (Figure 1A). This finding is consistent with the effect of Rhebl ko on mTORC1 activity in other brain regions [16]. Given the importance of $m$ TORC1 activity in hypothalamic neurons, particularly in POMC neurons, in the control of food intake, we examined the food intake of 3-week-old Rhebl ko mice. The results showed that the food intake by Rhebl ko mice was significantly reduced relative to the control mice (floxed Rhebl mice without $\mathrm{Cre})(\mathrm{N}=4)$ (Figure 1B). These data indicate that central Rhebl signaling plays a role in the control of food intake.

Figure 1. Genetic deletion of Rhebl in the brain disrupts food intake. (A) Western blotting showing that Rhebl and pS6 (Ser240/244) protein levels are reduced in Rhebl ko hypothalamus; (B) Histograms showing the drastic reduction of food intake in Rhebl ko mice compared to controls $(N=4)$. Results are averages of four independent animals. Data represent mean \pm SEM. *** $p<0.001$.

A

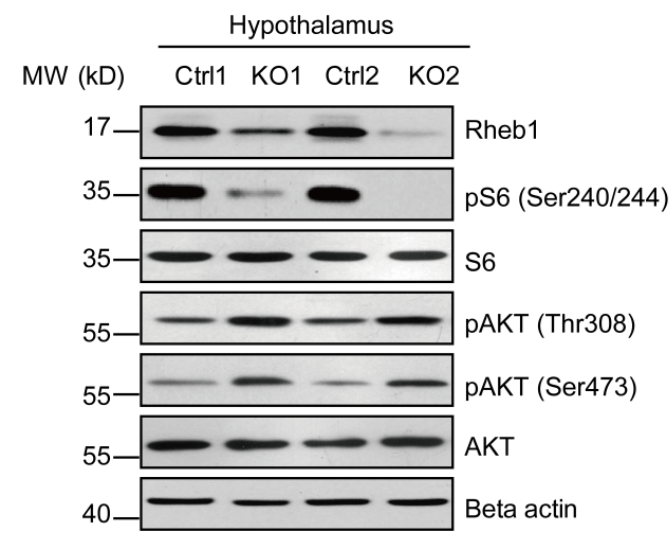

B

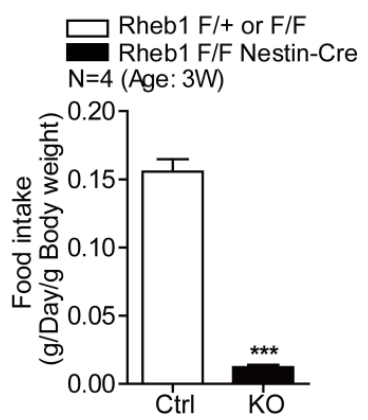




\subsection{Genetic Deletion of Rheb1 in the Brain Leads to Hypoglycemia}

Food intake is critical to maintain blood glucose level and energy homeostasis. We wondered if blood glucose level was altered in Rhebl ko mice. Blood glucose measurement shows that Rhebl ko mice had a much lower glucose level $(70 \mathrm{mg} / \mathrm{DL})$ than that in their control littermates $(150 \mathrm{mg} / \mathrm{DL})(N=4)$ (Figure 2A). To further examine the effect of Rhebl ko on energy homeostasis, we examined the gluconeogenesis in the liver where Rhebl/mTORC1 activity in hepatocytes was slightly increased (see below). Real-time PCR analysis revealed that the mRNA levels of two canonical gluconeogenesis genes, G6pc and Pckl [18], were increased in the liver of Rhebl ko mice in comparison with those in control livers (Figure 2B). This result suggested that gluconeogenesis was activated in Rhebl ko mice to synthesize glucose from non-carbohydrate precursors as part of homeostatic adaptation.

Figure 2. Genetic deletion of Rhebl in the brain leads to hypoglycemia. (A) Biochemical assays showing the robust reduction of blood glucose level in Rhebl ko mice compared controls $(N=4)$. Results are averages of four independent animals. Data represent mean \pm SEM. $* * p<0.01$; (B) Real-time PCR assays showing the increasing mRNA levels of G6pc and Pckl in livers of Rhebl ko mice $(N=3)$. Results are averages of three independent animals. Data represent mean \pm SEM. $* p<0.05, * * * p<0.001$.

A

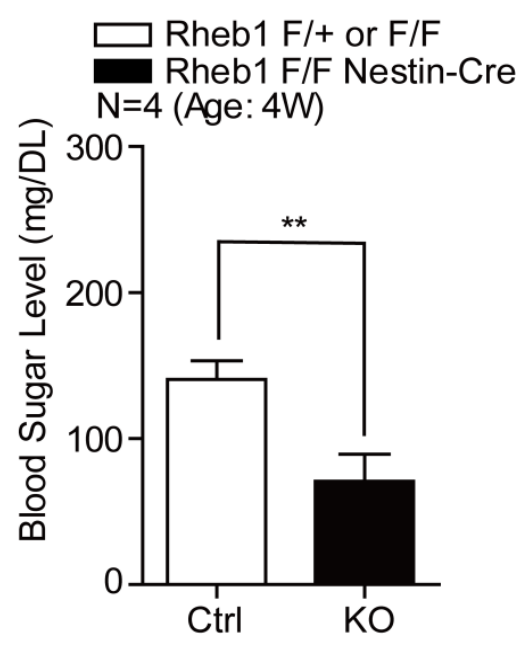

B

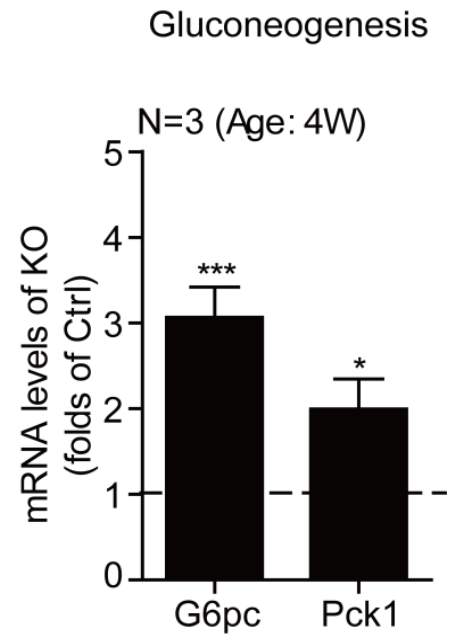

\subsection{Genetic Deletion of Rheb1 in the Brain Induces Ketogenesis in the Liver}

To further examine the adaptive metabolic changes as a result of altered Rheb1/mTORC1 activity in the brain, we assayed the ketogenesis in the liver of Rhebl ko mice. Ketogenesis is an essential component of metabolic adaptations to meet the energy demand of the brain. We examined ketogenesis in the liver of Rhebl ko mice and found that the ketogenesis was increased compared to the controls. This was demonstrated through increased levels of beta-hydroxybutyrate in serum of Rheb1 mice $(N=3)$ (Figure 3A) and increased mRNA levels of hydroxy-methyl-glutaryl coenzyme A synthase 2 (HMG-CoA synthase 2, Hmgcs 2$)$ and HMG-CoA lyase $(\mathrm{Hmgcl})(\mathrm{N}=3)$ - enzymes that are critical for ketogenesis in the liver $[19,20](N=3)$ (Figure 3B). Consistent with the mRNA increase of Hmgcs2, its protein level was also increased by $32.1 \%$ (Figure $3 \mathrm{C}, \mathrm{D}$ ). Increased ketogenesis is 
normally linked to increased lipid mobilization to provide free fatty acids for biosynthesis of ketone bodies in the liver. The majority of free fatty acids are generated as a consequence of the breakdown of triglycerides in adipose tissue and transferred to the liver through circulation for ketogenesis [21]. Therefore, we assayed the protein level of adipose triglyceride lipase ( $A \operatorname{tg} l)$ as an indicator of lipolysis. Western blotting revealed a significant increase of $A T G L$ protein level in the adipose tissue of Rhebl ko mice as compared to the controls $(N=3)$, suggesting increased lipid mobilization in Rhebl ko mice. Collectively, our data supports the notion that genetic deletion of Rhebl in the brain would increase adaptive lipid mobilization and ketogenesis to meet the energy demand of the brain.

Figure 3. Genetic deletion of Rhebl in the brain induces ketogenesis in the liver. (A) Biochemical assays showing increased blood ketone levels in 4-week-old Rhebl ko mice $(N=3)$. Results are averages of three independent animals. Data represent mean \pm SEM. * $p<0.05$; (B) Real-time PCR results showing the increasing levels of ketogenesis genes Hmgcs 2 and $\mathrm{Hmgcl}$ in livers of Rhebl ko mice $(N=3)$. Results are averages of three independent animals. Data represent mean $\pm \mathrm{SEM} . * p<0.05$; (C,D) Western blots and histograms showing the increasing of HMGCS2 protein level in livers of Rhebl ko mice $(N=3)$. Results are averages of three independent animals. Data represent mean \pm SEM. $* * p<0.01, * * * p<0.001$; (E,F) Western blots and histograms showing the increasing of $A T G L$ protein level in adipose of Rhebl ko mice $(N=3)$. Results are averages of three independent animals. Data represent mean \pm SEM. $* p<0.05$.

A

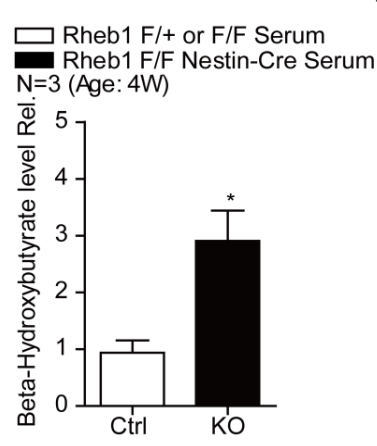

D

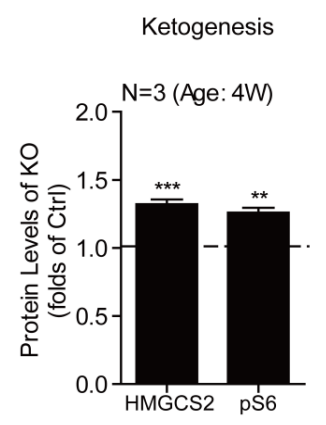

B

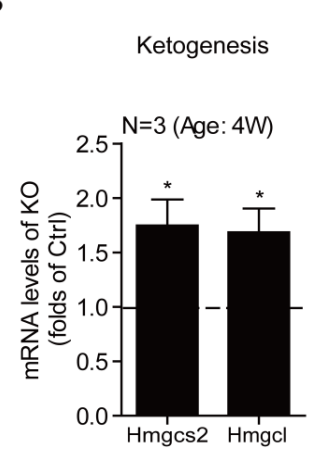

$\mathbf{E}$

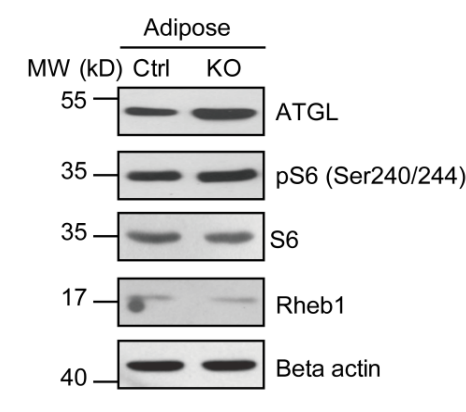

C

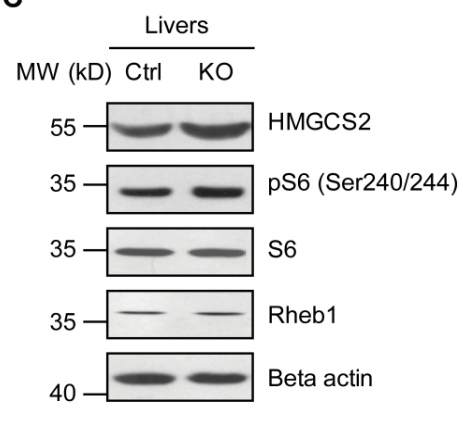

$\mathbf{F}$

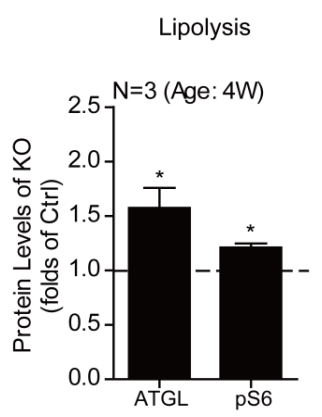

\subsection{Genetic Deletion of Rheb1 in the Brain Increases Liver Lipid Droplets and Beta Oxidation}

The excessive influx of free fatty acids to the liver as a result of increased lipid mobilization tends to increase the formation of lipid droplets in the liver [22]. In support of this notion, we found that the lipid accumulation in the form of lipid droplets was significantly increased in the liver of Rhebl ko 
mice. By oil red $\mathrm{O}$ staining, we found a significant increase of lipid droplets in the liver of 4-week-old Rheb1 ko mice relative to controls (Figure 4A). Moreover, real-time PCR results showed that the mRNA levels of genes that were critical for lipogenesis, such as Srebplc [23], Acl [24] and Fasn [23,24], were not increased in the liver of Rhebl ko mice $(N=3)$ (Figure 4B), suggesting that this lipid accumulation was not due to increased de novo lipogenesis in the liver. The fatty acids derived from adipose tissue are utilized by beta-oxidation to generate acetyl-CoA for energy production $[25,26]$. To investigate whether the beta-oxidation was activated to oxidize the excessive fatty acids in the liver of Rhebl ko mice, we assayed the mRNA and protein levels of genes that are critical for beta-oxidation. The results showed that the mRNA levels of Atgl [27], Ppara [28], Cpt1-a [29], Mlycd [30] and Mcad [31], were all increased in the liver of Rheb1 ko mice relative to controls $(N=3)$ (Figure 4C). Consistent with the increases in mRNA levels, protein levels for ATGL, PPAR $\alpha$ and CPT1-A were also increased as shown by Western blotting $(N=3)$ (Figure 4D,E). All these results support the notion that adipose-derived lipids are transferred to the liver for beta-oxidation and subsequent ketogenesis to meet the energy demand of the brain in Rhebl ko mice.

The present study uses genetic approaches to demonstrate a significant role of central Rhebl in the control of food intake and energy homeostasis. Because $m$ TORCl activity in hypothalamic neurons is critical for food intake $[4,15]$ and genetic deletion of Rhebl inhibits $m T O R C 1$ activity in hypothalamus (Figure 1A), it is probable that the reduced food intake and hypoglycemia in Rhebl ko mice is due to reduced $m$ TORC1 activity in hypothalamic neurons. To meet the energy demand of the brain, Rhebl ko mice develop adaptive changes in peripheral systems, such as lipid mobilization (Figure 3E), ketogenesis (Figure 3A) and gluconeogenesis (Figure 2B).

We noted that the increase in ketogenesis in the liver of Rhebl ko mice is not due to loss of Rhebl activity in hepatic cells, because we did not detect any significant change to Rhebl protein level in livers of Rhebl ko mice (Figure 3C). This non-cell autonomous effect of Rhebl is consistent with the report that Nestin is expressed in only hepatic stellate cells that account for only $5 \%$ of the total number of cells in the mouse liver [32]. Moreover, we found that mTORC1 activity was slightly increased in hepatic and adipose tissues of Rhebl ko mice (Figure 3C,E), suggesting that the increased ketogenesis and lipid mobilization in Rhebl ko mice is a secondary effect of loss of central Rhebl activity.

Animals develop multiple cellular mechanisms to control food intake to maintain energy homeostasis [1]. How food intake is regulated at the molecular level remains an unresolved question. Recent progress has focused on the importance of hypothalamic neurons in the control of food intake. As a central regulator of metabolisms, $m$ TOR not only controls the lipid and protein biosynthesis, but also manipulates the feeding behaviors. Several studies have showed that $m T O R$ signaling may serve as a negative regulator of food intake. For example, activation of hypothalamic mTORC1 signaling by central administration of leucine decreases food intake and body weight, and inhibition of mTORC1 signaling by rapamycin injection blunts leptin's anorectic effect [4]. This effect seems to be mediated by $S 6 K$, because $S 6 K$, which is a major downstream factor of $m T O R C 1$, also negatively regulates food intake [14]. However, contradictive evidence points out that activation of $m$ TORC1 by TSC ablation in hypothalamic POMC neurons increases food intake and body weight, while intracerebral rapamycin infusion restores it [15]. These controversial results raise the questions of how the mTOR pathway controls hypothalamic food intake center, anorexigenic or orexigenic. Of note, most of these studies are based on pharmacological or viral-mediated manipulations of $m$ TORC1 activity in vivo. Therefore, 
studies of genetically modified mouse models are necessary to clarify the role of $m T O R$ signaling in the control of food intake and energy homeostasis. Here, we examined the effect of Rhebl deletion in the brain on food intake and subsequent alterations to peripheral metabolism. Our results demonstrate that as a result of Rhebl deletion, $m$ TORCl activity in the hypothalamus is significantly reduced and the Rhebl ko mice exhibit significantly reduced food intake and hypoglycemia. To meet the energy demand of the brain, lipid mobilization is activated to increase the biosynthesis of ketone bodies in the liver.

Figure 4. Genetic deletion of Rhebl in the brain increases liver lipid droplets and beta oxidation. (A) Representative oil red O staining images showing increased number of lipid droplets in livers of Rhebl ko mice. Bar $25 \mu \mathrm{m}$; (B) Real-time PCR assays showing that hepatic lipogenesis genes Srebplc, Acl and Fasn are not increased in livers of Rhebl ko mice $(N=3)$. Results are averages of three independent animals. Data represent mean $\pm \mathrm{SEM}$. $* p<0.05$; (C) Real-time PCR assays showing the increasing of mRNA levels of beta-oxidation genes, Atgl, Ppara, Cpt1-a, Mlycd and Mcad in livers of Rhebl ko mice $(N=3)$. Results are averages of three independent animals. Data represent mean $\pm \mathrm{SEM}$. $* p<0.05, * * p<0.01$; (D,E) Western blots and histograms showing the increasing of protein levels of $A T G L, P P A R \alpha$ and $C P T 1-A$ in livers of Rhebl ko mice $(N=3)$. Results are averages of three independent animals. Data represent mean $\pm \mathrm{SEM}$. ${ }^{*} p<0.05$, $* * p<0.01$.

A

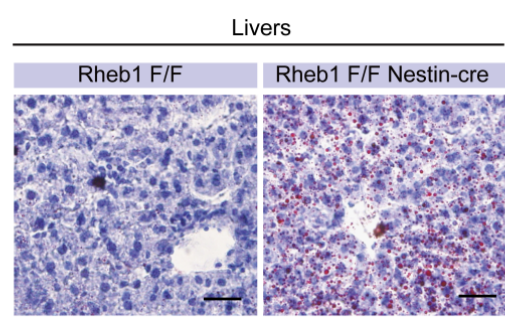

C

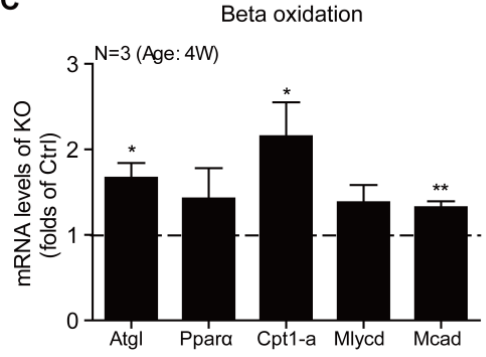

D
B
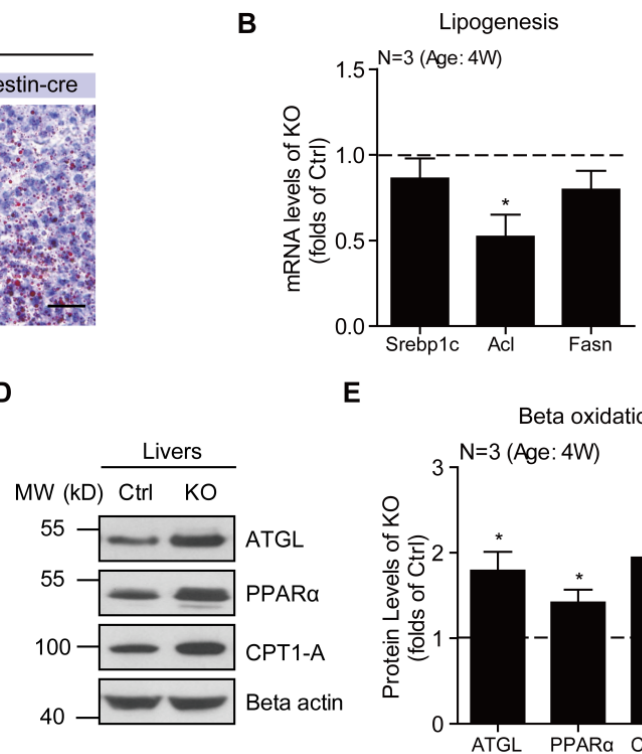

E

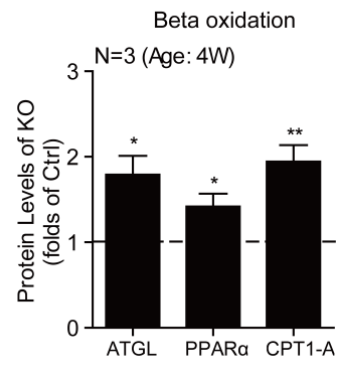

The central regulation of food intake is an immensely complex process involving the coordinated activities of multiple cerebral loci, such as the limbic system [33] and the cerebral cortex [34]. The neuronal processes from these loci project onto hypothalamic neurons and regulate appetite. Recent imaging studies indicate the awareness of hunger involves cortical functions [35]. Since the mTORC1 activity in the cortex is also affected in Rhebl ko mice, presumably, the awareness of hunger is also altered by Rhebl deletion. Although Rhebl has been widely accepted as a key activator of $m$ TORC1 signaling in vitro and in vivo, there may be additional $m$ TORCl-independent effects of Rhebl. Because genetic deletion of Rhebl in the brain increases $A K T$ activity by the mTORC1-S6K1-IRS feedback pathway, future studies will address whether increased $A K T$ activity contributes to the reduced food 
intake in Rhebl ko mice. Again, genetic deletion of $m$ TOR kinase or raptor may also help to further clarify whether the effect of Rhebl on food intake is mediated by mTORC1 signaling.

\section{Experimental Section}

\subsection{Reagents and Antibodies}

The biochemical kit of beta-hydroxybutyrate was purchased from Biovision (Milpitas, CA, USA). The following antibodies, ATGL and pS6 (S240/244), were from Cell Signaling Technology (Danvers, MA, USA). Anti- $\beta$-actin was purchased from Millipore (Billerica, MA, USA). And CPT1-A antibody was from Abcam (Cambridge, UK). PPAR $\alpha$ was from Thermo Scientific (Rockford, IL, USA), and HMGCS2 from LifeSpan BioScience (Seattle, WA, USA). Anti-Rheb1 antibody has been described previously [16].

\subsection{Animals}

Rhebl ko mice were generated by breeding floxed Rhebl mice with Nestin-cre deleter mice, and genotyped as previously described [16]. Both the Rhebl ko and control littermates were housed in environmentally controlled conditions with a 12-hour light/dark cycle, and fed normal chow. All mouse work was done in accordance with the Animal Care and Use Committee guidelines of Sichuan University West-China Hospital.

\subsection{Blood Glucose Measurement}

Blood glucose levels were measured in a drop of blood obtained from tails of 4-week-old Rheb1 ko mice and control littermates at 10:00 a.m. in the fed state. The blood glucose analysis was performed using the Roche Accu-Chek Active System (Roche Diagnostics, Mannheim, Germany).

\subsection{Western Blotting}

Mouse adipose, liver and hypothalamus extracts were made with lysis buffer (2\% SDS with protease and phosphatase inhibitors). Same amounts of proteins were loaded into SDS-PAGE gels and blotted with various antibodies, according to standard Western blotting procedures.

\subsection{RNA Extraction and Real-Time PCR Assay}

Total RNAs were extracted from tissues using Trizol reagent (Invitrogen, Carlsbad, CA, USA). RNA was subjected to reverse transcription with reverse transcriptase according to the manufacturer's instructions (Fermentas, Glen Burnie, MD, USA). Quantitative real-time PCR was performed using the Bio-Rad iQ5 system (Bio-Rad, Hercules, CA, USA), and the relative gene expression was normalized to internal control as beta-actin. Primer sequences for SYBR Green probes of target genes were as follows:

G6pc: 5'-GTGTCCGTGATCGCAGACC-3' and 5'-GACGAGGTTGAGCCAGTCTC-3'; Pck1: 5'-T TGAGAAAGCGTTCAATGCCA-3' and 5'-CACGTAGGGTGAATCCGTCAG-3'; 
Hmgcs2: 5'-ATA CCACCAACGCCTGTTATGG-3' and 5'-CAATGTCACCACAGACCACCAG-3'; Hmgcl: 5'-ACTA CCCAGTCCTGACTCCAA-3' and 5'-TAGAGCAGTTCGCGTTCTTCC-3'; Srebplc: 5'-GGAGCC ATGGATTGCACATT-3' and 5'-GCTTCCAGAGAGGAGGCCAG-3'; Acl: 5'-GAAGCTGACCTT GCTGAACC-3' and 5'-CTGCCTCCAATGATGAGGAT-3'; Fasn: 5'-TGGGTTCTAGCCAGCAGA GT-3' and 5'-ACCACCAGAGACCGTTATGC-3'; Atgl: 5'-TGTGGCCTCATTCCTCCTAC-3' and 5'-TGCTGGATGTTGGTGGAGCT-3'; Ppara: 5'-TGTTTGTGGCTGCTATAATTTGC-3' and 5'-GCAACTTCTCAATGTAGCCTATGTTT-3'; Cpt1-a: 5'-GGAGAGAATTTCATCCACTTCCA-3' and 5'-CTTCCCAAAGCGGTGTGAGT-3'; Mlycd: 5'-GCACGTCCGGGAAATG AAC-3' and 5'-GCCTCACACTCGCTGATCTT-3'; Mcad: 5'-TTTCGAAGACGTCAGAGTGC-3' and 5'-TGCGACTGTAGGTCTGGTTC-3'; Beta-actin: 5'-GAGACCTTCAACACCCCAGC-3' and 5'-ATGTCACGCACGATTTCCC-3'.

\section{6. $\beta$-Hydroxybutyrate Assay}

Serum samples from Rhebl ko or control mice (fed normally) were collected into a 96-well plate by retroorbital bleeding according to standard procedure. The procedure was performed at approximately same time on the days when the blood glucose assay was carried out. Beta-hydroxybutyrate levels were measured using a commercial diagnostic kit of Biovision (Catalog. \#K632-100, Milpitas, CA, USA) according to the manufacturer's instruction.

\subsection{Oil Red O Staining}

Standard Oil Red O staining was performed on frozen liver sections to evaluate tissue lipid content. After sacrifice, fresh livers were frozen immediately and subsequently cut into $10 \mu \mathrm{m}$ sections by Cryostat Microtome. The staining solution was prepared by dissolving $0.5 \mathrm{~g}$ oil-red-O (Sigma, St. Louis, MO, USA) in $100 \mathrm{~mL}$ of isopropanol; $60 \mathrm{~mL}$ of this solution was mixed with $40 \mathrm{~mL}$ of distilled water. After $1 \mathrm{~h}$ at room temperature the staining solution was filtered and added to liver slices for $15 \mathrm{~min}$. The staining solution was then removed and liver slices were washed twice with distilled water.

\subsection{Food Intake Assay}

For food intake experiments, three-week-old Rhebl ko mice and control littermates were separated and each mouse was raised with chow (10 g/day) in a single cage. After $24 \mathrm{~h}$, all the remainder food was collected and quantified. The experiments lasted for five days. Then the mass of intake food was normalized to mouse body weight for statistical analysis.

\subsection{Statistical Analysis}

All results of Western blotting, real-time PCR, and food intake across time were presented as mean \pm SEM from a minimum of three or four independent experiments. Data from Western-blots were analyzed by ImageJ software (U.S. National Institutes of Health (NIH), Bethesda, MD, USA). $P$ values were calculated using the Student's $t$ test for normally distributed data, and the value $0.05(*)$, $0.01(* *)$ and $0.001(* * *)$ was assumed as the level of significance for the statistic tests carried out. 


\section{Conclusions}

In conclusion, our work highlights an important role of central Rhebl in euglycemia and energy homeostasis in animals, which is mostly likely mediated by mTORC1 signaling. Our study sets the stage to examine how Rheb1/mTORCl activity in hypothalamic neurons controls food intake and alters peripheral metabolism.

\section{Acknowledgments}

This study was supported by the grant from National 973 Basic Research Program of China (2009CB941400) and Sichuan Science and Technology Support Program of China (2012SZ0152).

\section{Conflicts of Interest}

The authors declare no conflict of interest.

\section{References}

1. Coupe, B.; Grit, I.; Hulin, P.; Randuineau, G.; Parnet, P. Postnatal growth after intrauterine growth restriction alters central leptin signal and energy homeostasis. PLoS One 2012, 7, e30616.

2. Hardie, D.G.; Ross, F.A.; Hawley, S.A. AMPK: A nutrient and energy sensor that maintains energy homeostasis. Nat. Rev. Mol. Cell Biol. 2012, 13, 251-262.

3. Pimentel, G.D.; Ropelle, E.R.; Rocha, G.Z.; Carvalheira, J.B. The role of neuronal AMPK as a mediator of nutritional regulation of food intake and energy homeostasis. Metab. Clin. Exp. 2013, 62, 171-178.

4. Cota, D.; Proulx, K.; Smith, K.A.; Kozma, S.C.; Thomas, G.; Woods, S.C.; Seeley, R.J. Hypothalamic mTOR signaling regulates food intake. Science 2006, 312, 927-930.

5. Wiczer, B.M.; Thomas, G. The role of the mTOR pathway in regulating food intake. Curr. Opin. Drug Discov. Dev. 2010, 13, 604-612.

6. Woods, S.C.; Seeley, R.J.; Cota, D. Regulation of food intake through hypothalamic signaling networks involving mTOR. Ann. Rev. Nutr. 2008, 28, 295-311.

7. Laplante, M.; Sabatini, D.M. An emerging role of mTOR in lipid biosynthesis. Curr. Biol. 2009, 19, R1046-R1052.

8. Yecies, J.L.; Manning, B.D. Transcriptional control of cellular metabolism by mTOR signaling. Cancer Res. 2011, 71, 2815-2820.

9. Braun, S.; Bitton-Worms, K.; LeRoith, D. The link between the metabolic syndrome and cancer. Int. J. Biol. Sci. 2011, 7, 1003-1015.

10. Jacinto, E.; Facchinetti, V.; Liu, D.; Soto, N.; Wei, S.; Jung, S.Y.; Huang, Q.; Qin, J.; Su, B. SIN1/MIP1 maintains rictor-mTOR complex integrity and regulates Akt phosphorylation and substrate specificity. Cell 2006, 127, 125-137.

11. Sarbassov, D.D.; Guertin, D.A.; Ali, S.M.; Sabatini, D.M. Phosphorylation and regulation of Akt/PKB by the rictor-mTOR complex. Science 2005, 307, 1098-1101. 
12. Nobukuni, T.; Joaquin, M.; Roccio, M.; Dann, S.G.; Kim, S.Y.; Gulati, P.; Byfield, M.P.; Backer, J.M.; Natt, F.; Bos, J.L.; et al. Amino acids mediate mTOR/raptor signaling through activation of class 3 phosphatidylinositol 3OH-kinase. Proc. Natl. Acad. Sci. USA 2005, 102, $14238-14243$.

13. Garami, A.; Zwartkruis, F.J.; Nobukuni, T.; Joaquin, M.; Roccio, M.; Stocker, H.; Kozma, S.C.; Hafen, E.; Bos, J.L.; Thomas, G. Insulin activation of Rheb, a mediator of mTOR/S6K/4E-BP signaling, is inhibited by TSC1 and 2. Mol.Cell 2003, 11, 1457-1466.

14. Blouet, C.; Ono, H.; Schwartz, G.J. Mediobasal hypothalamic p70 S6 kinase 1 modulates the control of energy homeostasis. Cell Metab. 2008, 8, 459-467.

15. Yang, S.B.; Tien, A.C.; Boddupalli, G.; Xu, A.W.; Jan, Y.N.; Jan, L.Y. Rapamycin ameliorates age-dependent obesity associated with increased mTOR signaling in hypothalamic POMC neurons. Neuron 2012, 75, 425-436.

16. Zou, J.; Zhou, L.; Du, X.X.; Ji, Y.; Xu, J.; Tian, J.; Jiang, W.; Zou, Y.; Yu, S.; Gan, L.; et al. Rheb1 is required for mTORC1 and myelination in postnatal brain development. Dev. Cell 2011, 20, 97-108.

17. Tronche, F.; Kellendonk, C.; Kretz, O.; Gass, P.; Anlag, K.; Orban, P.C.; Bock, R.; Klein, R.; Schutz, G. Disruption of the glucocorticoid receptor gene in the nervous system results in reduced anxiety. Nat. Genet. 1999, 23, 99-103.

18. Ozcan, L.; Wong, C.C.; Li, G.; Xu, T.; Pajvani, U.; Park, S.K.; Wronska, A.; Chen, B.X.; Marks, A.R.; Fukamizu, A.; et al. Calcium signaling through CaMKII regulates hepatic glucose production in fasting and obesity. Cell Metab. 2012, 15, 739-751.

19. Kostiuk, M.A.; Keller, B.O.; Berthiaume, L.G. Palmitoylation of ketogenic enzyme HMGCS2 enhances its interaction with PPARalpha and transcription at the Hmgcs2 PPRE. FASEB J. 2010, 24, 1914-1924.

20. Pie, J.; Lopez-Vinas, E.; Puisac, B.; Menao, S.; Pie, A.; Casale, C.; Ramos, F.J.; Hegardt, F.G.; Gomez-Puertas, P.; Casals, N. Molecular genetics of HMG-CoA lyase deficiency. Mol. Genet. Metab. 2007, 92, 198-209.

21. Kolditz, C.I.; Langin, D. Adipose tissue lipolysis. Curr. Opin. Clin. Nutr. Metab. Care 2010, 13, 377-381.

22. Foretz, M.; Ancellin, N.; Andreelli, F.; Saintillan, Y.; Grondin, P.; Kahn, A.; Thorens, B.; Vaulont, S.; Viollet, B. Short-term overexpression of a constitutively active form of AMP-activated protein kinase in the liver leads to mild hypoglycemia and fatty liver. Diabetes $\mathbf{2 0 0 5}$, 54, 1331-1339.

23. Porstmann, T.; Santos, C.R.; Griffiths, B.; Cully, M.; Wu, M.; Leevers, S.; Griffiths, J.R.; Chung, Y.L.; Schulze, A. SREBP activity is regulated by mTORC1 and contributes to Akt-dependent cell growth. Cell Metab. 2008, 8, 224-236.

24. Bobrovnikova-Marjon, E.; Hatzivassiliou, G.; Grigoriadou, C.; Romero, M.; Cavener, D.R.; Thompson, C.B.; Diehl, J.A. PERK-dependent regulation of lipogenesis during mouse mammary gland development and adipocyte differentiation. Proc. Natl. Acad. Sci. USA 2008, 105, 16314-16319.

25. Lopes-Cardozo, M.; van den Bergh, S.G. Ketogenesis in isolated rat liver mitochondria. III. Relationship with the rate of beta-oxidation. Biochim. Biophys. Acta 1974, 357, 53-62. 
26. Serviddio, G.; Giudetti, A.M.; Bellanti, F.; Priore, P.; Rollo, T.; Tamborra, R.; Siculella, L.; Vendemiale, G.; Altomare, E.; Gnoni, G.V. Oxidation of hepatic carnitine palmitoyl transferase-I (CPT-I) impairs fatty acid beta-oxidation in rats fed a methionine-choline deficient diet. PLoS One 2011, 6, e24084.

27. Chakrabarti, P.; Kandror, K.V. Adipose triglyceride lipase: A new target in the regulation of lipolysis by insulin. Curr. Diabetes Rev. 2011, 7, 270-277.

28. El Kebbaj, Z.; Andreoletti, P.; Mountassif, D.; Kabine, M.; Schohn, H.; Dauca, M.; Latruffe, N.; El Kebbaj, M.S.; Cherkaoui-Malki, M. Differential regulation of peroxisome proliferator-activated receptor (PPAR)-alpha1 and truncated PPARalpha2 as an adaptive response to fasting in the control of hepatic peroxisomal fatty acid beta-oxidation in the hibernating mammal. Endocrinology 2009, 150, 1192-1201.

29. Gaidhu, M.P.; Fediuc, S.; Ceddia, R.B. 5-Aminoimidazole-4-carboxamide-1- $\beta$-D-ribofuranosideinduced AMP-activated protein kinase phosphorylation inhibits basal and insulin-stimulated glucose uptake, lipid synthesis, and fatty acid oxidation in isolated rat adipocytes. J. Biol. Chem. 2006, 281, 25956-25964.

30. Derdak, Z.; Villegas, K.A.; Harb, R.; Wu, A.M.; Sousa, A.; Wands, J.R. Inhibition of p53 attenuates steatosis and liver injury in a mouse model of non-alcoholic fatty liver disease. J. Hepatol. 2012, 58, 785-791.

31. Hanley, P.J.; Gopalan, K.V.; Lareau, R.A.; Srivastava, D.K.; von Meltzer, M.; Daut, J. $\beta$-Oxidation of 5-hydroxydecanoate, a putative blocker of mitochondrial ATP-sensitive potassium channels. J. Physiol. 2003, 547, 387-393.

32. Niki, T.; Pekny, M.; Hellemans, K.; Bleser, P.D.; Berg, K.V.; Vaeyens, F.; Quartier, E.; Schuit, F.; Geerts, A. Class VI intermediate filament protein nestin is induced during activation of rat hepatic stellate cells. Hepatology 1999, 29, 520-527.

33. Fukamauchi, F.; Aihara, O.; Kusakabe, M. Reduced mRNA expression of neuropeptide Y in the limbic system of tenascin gene disrupted mouse brain. Neuropeptides 1998, 32, 265-268.

34. Tacconi, M.T.; Lligona, L.; Salmona, M.; Pitsikas, N.; Algeri, S. Aging and food restriction: Effect on lipids of cerebral cortex. Neurobiol. Aging 1991, 12, 55-59.

35. Lassman, D.J.; McKie, S.; Gregory, L.J.; Lal, S.; D’Amato, M.; Steele, I.; Varro, A.; Dockray, G.J.; Williams, S.C.; Thompson, D.G. Defining the role of cholecystokinin in the lipid-induced human brain activation matrix. Gastroenterology 2010, 138, 1514-1524.

(C) 2014 by the authors; licensee MDPI, Basel, Switzerland. This article is an open access article distributed under the terms and conditions of the Creative Commons Attribution license (http://creativecommons.org/licenses/by/3.0/). 